\title{
Particle Learning for Fat-tailed Distributions ${ }^{1}$
}

\author{
Hedibert F. Lopes and Nicholas G. Polson \\ University of Chicago Booth School of Business
}

\begin{abstract}
It is well-known that parameter estimates and forecasts are sensitive to assumptions about the tail behavior of the error distribution. In this paper we develop an approach to sequential inference that also simultaneously estimates the tail of the accompanying error distribution. Our simulation-based approach models errors with a $t_{\nu}$-distribution and, as new data arrives, we sequentially compute the marginal posterior distribution of the tail thickness. Our method naturally incorporates fat-tailed error distributions and can be extended to other data features such as stochastic volatility. We show that the sequential Bayes factor provides an optimal test of fat-tails versus normality. We provide an empirical and theoretical analysis of the rate of learning of tail thickness under a default Jeffreys prior. We illustrate our sequential methodology on the British pound/US dollar daily exchange rate data and on data from the 2008-2009 credit crisis using daily S\&P500 returns. Our method naturally extends to multivariate and dynamic panel data.
\end{abstract}

JEL: C01, C11, C15, C16, C22, C58.

Keywords: Bayesian Inference, MCMC, Kullback-Leibler, Dynamic Panel Data, Credit Crisis.

\footnotetext{
${ }^{1}$ Corresponding author: Hedibert Freitas Lopes. The University of Chicago Booth School of Business. 5807 South Woodlawn Avenue, Chicago, IL 60637, USA. Phone number: +1 (773) 8345458. Fax number: +1 (773) 702-0458. E-mail: hlopes@chicagobooth.edu.
} 


\section{Introduction}

Fat-tails are an important statistical property of time series prevalent in many fields, particularly economics and finance. Fat-tailed error distributions were initially introduced by Edgeworth (1888) and explored further by Jeffreys (1961) who once remarked that "... all data are $t_{4}$ ". They can be incorporated into dynamic models as latent variable scale mixtures of normals (Carlin, Polson and Stoffer, 1992, FrühwirthSchnatter, 2006). In this paper, we develop a simulation-based sequential inference procedure for estimating the tail behavior of a time series using the $t_{\nu}$-distribution. This family is attractive for this purpose due to its flexibility with normality $(\nu=\infty)$ and Cauchy $(\nu=1)$ errors as special cases. Our method complements the existing literature by estimating the set of sequential posterior distributions $p\left(\nu \mid y^{t}\right)$ for data $y^{t}=\left(y_{1}, \ldots, y_{t}\right)$ and $t=1, \ldots, T$, as opposed to MCMC which estimates $\nu$ given the full data history $p\left(\nu \mid y^{T}\right)$ (see Geweke, 1993, Eraker, Jacquier and Polson (JPR), 1998, Jaquier, Polson and Rossi, 2004 and Fonseca, Ferreira and Migon, 2008). In other words, our methodology allows the researcher to estimate and update not only model parameters but also the tail-thickness of the error distribution as new data arrives.

The novel feature of our approach are the on-line estimates of the tail thickness of the error distribution using the marginal posterior distribution of the degrees of freedom parameter $\nu$. Our method is based on particle learning (PL, see Carvalho et al., 2010, and Lopes et al., 2010). We analyze two cases in detail: in the first observations $y_{t}$ follow the independent and identically distributed standard $t_{\nu^{-}}$ distribution, i.e. $y_{t} \sim t_{\nu}(0,1)$ (iid- $t$ case), and in the second observations follow a non-identically distributed stochastic volatility model with fat-tails (SV-t case), i.e. $y_{t} \mid h_{t} \sim t_{\nu}\left(0, \exp \left\{h_{t}\right\}\right)$ are conditionally independent given the $T$-dimensional latent 
vector of log-volatilities $h^{T}=\left(h_{1}, \ldots, h_{T}\right)$, see JPR (2004) and Chib, Nardari and Shephard (2002).

Our posterior distribution $p\left(\nu \mid y^{t}\right)$ on the tail thickness is sensitive to the choice of prior distribution, $p(\nu)$. We model the prior on the degrees of freedom $\nu$ using a default Jeffreys prior (Fonseca et al., 2008). In this setting, we show that the Jeffreys prior has desirable properties. Primarily, it reduces bias for estimating the tail thickness in small sized data sets. Moreover, it is well known that more data helps to discriminate similar error distributions. Hence a priori we know that we will need a larger dataset to discriminate a $t_{20}$-distribution from a normal distribution than a $t_{4}$-distribution from a normal. We develop a metric based on the asymptotic Kullback-Liebler rate of learning of tail thickness that can guide the amount of data required to discriminate two error distributions. Given the observed data, we then develop an empirical and theoretical analysis of the sequential Bayes factor provides which provides the optimal test of normality versus fat-tails in our sequential context.

Recent estimation approaches for fat-tails use approximate latent Gaussian models (McCausland, 2008). We use the traditional data augmentation with a vector of latent scale variables $\lambda_{t}$ to avoid evaluating the likelihood (a $T$-dimensional integral). We develop a particle learning algorithm for sampling from the sequential set of joint posterior distributions $p\left(\lambda_{t}, \nu \mid y^{t}\right)$, for the iid- $t$ case, and from $p\left(\lambda_{t}, h_{t}, \nu \mid y^{t}\right)$, for the SV- $t$ case, for $t=1, \ldots, T$. The marginal posterior distribution $p\left(\nu \mid y^{t}\right)$ provides estimates of the tail-thickness of the error distribution. The purpose for developing new estimation methods is apparent from a remark of Smith (2000) who warns that the likelihood for non-Gaussian models can have several local maxima, be very skewed, or have modes on the boundary of the parameter space, making estimating tail behavior a complex statistical problem. 
The rest of the paper is outlined as follows. Section 2 describes how to sequentially learn the tail of the $t_{\nu}$-distribution under iid- $t$ and SV- $t$ models. Section 3 discusses our particle learning implementation. We focus on using a default Jeffreys prior, showing that this has a number of desirable properties when learning the fat-tailed error distribution with finite samples. Section 4 provides an analysis of the sequential Bayes factor for testing normality versus fat-tails. Section 5 provides our empirical analysis and comparisons including an analysis of the British pound and US dollar daily exchange rate and daily S\&P500 returns from the credit crisis. Jacquier et al. (2004) apply MCMC methods to the SV-t model to daily exchange rate on the British pound versus the US dollar and we provide a sequential analysis for comparative purposes. Finally, Section 6 concludes.

\section{$2 \quad t_{\nu}$-distributed errors}

Consider data $y^{t}=\left(y_{1}, \ldots, y_{t}\right)$ arising from a fat-tailed $t_{\nu}$-distribution. The data are observed on-line and we wish our estimation procedure to take this into account. Given a prior distribution $p(\nu)$, the aim is to compute a set of sequential marginal posterior distributions $p\left(\nu \mid y^{t}\right)$ which are given by Bayes rule

$$
p\left(\nu \mid y^{t}\right)=\frac{p\left(y^{t} \mid \nu\right) p(\nu)}{\int p\left(y^{t} \mid \nu\right) p(\nu) d \nu} .
$$

The marginal likelihood is given by $p\left(y^{t} \mid \nu\right)$. In an iid setting, this likelihood is simply $p\left(y^{t} \mid \nu\right)=\prod_{i=1}^{t} p\left(y_{i} \mid \nu\right)$, a product of marginals. In the SV-t setting, it is more complicated and requires integrating out the unobserved $t$-dimensional vector of log- 
volatilities $h^{t}=\left(h_{1}, \ldots, h_{t}\right)$, namely

$$
p\left(y^{t} \mid \nu\right)=\int \prod_{i=1}^{t} p\left(y_{i} \mid h_{i}, \nu\right) p\left(h^{t}\right) d h^{t}
$$

where $p\left(y_{i} \mid h_{i}, \nu\right) \sim t_{\nu}\left(0, \exp \left\{h_{i}\right\}\right)$. On advantage of particle methods is that this computation will naturally occur within the procedure. Our task is to provide sequential inference for the degrees of freedom or tail thickness parameter, $\nu$, via the set of marginal posterior distributions $p\left(\nu \mid y^{t}\right)$, for $t=1, \ldots, T$. To do this, we will first use a standard data augmentation and then provide a sequential Monte Carlo algorithm to sample from $p\left(\lambda_{t}, \nu \mid y^{t}\right)$ which we now describe for the iid- $t$ and SV- $t$ models.

\subsection{The iid- $t$ model}

Consider iid observations $y_{t}$, for $t=1, \ldots, T$, from a fat-tailed location-scale model

$$
y_{t}=\mu+\sigma \eta_{t} \quad \text { where } \eta_{t} \stackrel{\text { iid }}{\sim} t_{\nu}(0,1)
$$

Data augmentation uses a scale mixture of normals representation by writing $\eta_{t}$ in two steps: i) $\eta_{t}=\sqrt{\lambda_{t}} \epsilon_{t}$ and ii) $\lambda_{t} \stackrel{\text { iid }}{\sim} I G(\nu / 2, \nu / 2)$, where $I G$ denotes the inverse gamma distribution. The marginal data distribution, integrating out $\lambda_{t}$, is then the fat-tailed $t_{\nu}$-distribution $p\left(y_{t} \mid \nu, \mu, \sigma^{2}\right) \sim t_{\nu}\left(\mu, \sigma^{2}\right)$, where $\sigma^{2}$ can be interpreted as a scale parameter. This leads to a model

$$
y_{t}=\mu+\sigma \sqrt{\lambda_{t}} \epsilon_{t} \quad \text { where }\left(\lambda_{t} \mid \nu\right) \stackrel{\text { iid }}{\sim} I G(\nu / 2, \nu / 2) \text { and } \epsilon_{t} \stackrel{\text { iid }}{\sim} N(0,1)
$$

By doing so, we have created a conditionally dynamic Gaussian model (FrühwirthSchnatter, 2006). For a given $\nu$, estimation of the other parameters results in a 
mixture Kalman filter algorithm (Chen and Liu, 2000, Carvalho et al., 2010). We will focus on extending this to incorporate learning about $\nu$. These specifications lead to a likelihood function $p\left(y \mid \mu, \sigma^{2}, \nu\right)$ of the form

$$
p\left(y \mid \mu, \sigma^{2}, \nu\right)=\prod_{t=1}^{T} \frac{\Gamma\left(\frac{\nu+1}{2}\right)}{\sqrt{\nu} \Gamma\left(\frac{\nu}{2}\right)}\left[1+\frac{1}{\nu}\left(\frac{y_{t}-\mu}{\sigma}\right)^{2}\right]^{-\frac{\nu+1}{2}}
$$

with marginal distribution $p\left(y_{t} \mid \nu\right)=\int p\left(y_{t} \mid \nu, \mu, \sigma^{2}\right) p\left(\mu, \sigma^{2}\right) d \mu d \sigma^{2}$. Fonseca et al. (2008) make the important observation that the marginal likelihood for $\nu$ becomes unbounded as $\nu \rightarrow \infty$ and the maximum likelihood estimator is not well-defined. This leads us to further develop an approach based on prior regularization, namely that the degrees of freedom parameter $\nu$ is random with a prior distribution $p(\nu)$ which we further discuss in Section 2.3.

Inference on the parameters $\left(\mu, \sigma^{2}\right)$ is not the focus of our study and for simplicity we assume that either they are known quantities or taken from a standard diffuse prior, $p(\mu) \propto 1$ and inverse-gamma prior $\sigma^{2} \sim I G\left(n_{0} / 2, n_{0} \sigma_{0}^{2} / 2\right)$ given hyperparameters $n_{0}$ and $\sigma_{0}^{2}$. These parameters control, respectively, the shape and the location of the distribution.

\subsection{The SV- $t$ model}

A common model of time-varying volatility is the stochastic volatility model with fat-tails (SV-t) for returns and volatility (see Lopes and Polson, 2010a, for a recent review). The basic SV model is specified by evolution dynamic

$$
\begin{array}{rlrl}
y_{t} & =\exp \left\{h_{t} / 2\right\} \epsilon_{t} & \epsilon_{t} \stackrel{\mathrm{iid}}{\sim} N(0,1) \\
h_{t}=\alpha+\beta h_{t-1}+\tau u_{t} & u_{t} \stackrel{\mathrm{iid}}{\sim} N(0,1) .
\end{array}
$$


The fat-tailed SV-t is obtained by adding an extra random scale parameter $\lambda_{t}$ and, as described in the conditionally iid setting, is equivalent to assuming that $\epsilon_{t} \sim t_{\nu}(0,1)$ (see, for example, JPR, 2004). The model can then be expressed as

$$
\begin{array}{ll}
y_{t}=\exp \left\{h_{t} / 2\right\} \sqrt{\lambda_{t}} \epsilon_{t} & \epsilon_{t} \stackrel{\mathrm{iid}}{\sim} N(0,1) \\
h_{t}=\alpha+\beta h_{t-1}+\tau u_{t} & u_{t} \stackrel{\mathrm{iid}}{\sim} N(0,1) \\
\lambda_{t} \stackrel{\mathrm{iid}}{\sim} I G(\nu / 2, \nu / 2) . &
\end{array}
$$

The parameter $\beta$ is the persistence of the volatility process and $\tau^{2}$ the volatility of the log-volatility. Estimation of these parameters will be greatly affected by the fat-tail error assumptions which in turn will affect predicting price and volatility (see, for example, Jacquier and Polson, 2000).

To complete the model specification, we need a prior distribution for the parameters $\left(\alpha, \beta, \tau^{2}\right)$ given $\nu$. For simplicity, we take a conditionally conjugate normal-

inverse-gamma-type prior. Specifically, $(\alpha, \beta) \mid \tau^{2} \sim N\left(b_{0}, \tau^{2} B_{0}\right)$ and $\tau^{2} \sim I G\left(c_{0}, d_{0}\right)$, for known hyper-parameters $b_{0}, B_{0}, c_{0}$ and $d_{0}$. This conditionally conjugate structure will aid in the development of our particle learning algorithm as it leads to conditional sufficient statistics. Non-conjugate prior specifications can also be handled in our framework, see Lopes et al. (2010) for further discussion.

\subsection{Priors on $\nu$}

In the models considered so far, an important modeling assumption is the regularization penalty $p(\nu)$ on the tail thickness. A default Jeffreys-style prior was developed by Fonseca et al. (2008) and, we will see, with a number of desirable properties particularly when learning a fat-tail (e.g., a $t_{4}$-distribution) from a finite dataset. The 
default Jeffreys prior for $\nu$ takes the form

$$
p(\nu)=\frac{1}{\sigma}\left(\frac{\nu}{\nu+3}\right)^{1 / 2}\left\{\psi^{\prime}\left(\frac{\nu}{2}\right)-\psi^{\prime}\left(\frac{\nu+1}{2}\right)-\frac{2(\nu+3)}{\nu(\nu+1)^{2}}\right\}^{1 / 2}
$$

where $\psi^{\prime}(a)=d\{\psi(a)\} / d a$ and $\psi(a)=d\{\log \Gamma(a)\} / d a$ are the trigamma and digamma functions, respectively. The interesting feature of this prior is its behavior as $\nu$ goes to infinity and it has polynomial tails of the form $p(\nu) \sim \nu^{-4}$. This is in contrast to commonly used priors such as Fernandez and Steel (1999) and Geweke (1993) who essentially specify priors with exponential tails of the form $\nu \exp \{-\lambda \nu\}$, for a subjectively chosen hyper-parameter, $\lambda$. In this case, the tail of the prior decays rather fast for large values of $\nu$ and assessing the degree of tail thickness can require prohibitively large samples.

In our empirical analysis we will show how this prior reduces bias in the posterior mean $E\left(\nu \mid y^{t}\right)$ and also how it helps discriminate a fat-tailed $t_{4}$-distribution from normality. On the other hand, the flat uniform prior suffers from placing too much mass on high values of $\nu$ - which are close to normality - making the inference problem harder for finite samples.

\section{Particle learning for fat-tails}

We now provide a discussion of particle learning with particular reference to estimating fat-tails. Sequential Bayesian computation requires calculation of a set of posterior distributions $p\left(\nu \mid y^{t}\right)$, for $t=1, \ldots, T$, where $y^{t}=\left(y_{1}, \ldots, y_{t}\right)$. Carvalho et al. (2010) and Lopes et al. (2010) present a sequential simulation strategy to both $p\left(\nu \mid y^{t}\right)$ and $p\left(y^{t}\right)$ based on a resample-sampling framework called particle learning $(\mathrm{PL})$. 
Central to PL is the creation of a essential state vector $Z_{t}$ to be tracked sequentially. We assume that this vector is conditionally sufficient for the parameter of interest; so that $p\left(\nu \mid Z_{t}\right)$ is either available in closed-form or can easily be sampled from. More precisely, given samples $\left\{Z_{t}^{(i)}\right\}_{i=1}^{N} \sim p\left(Z_{t} \mid y^{t}\right)$ and a Rao-Blackwellised identity, then a simple mixture approximation to the set of posteriors is given by

$$
p^{N}\left(\nu \mid y^{t}\right)=\frac{1}{N} \sum_{i=1}^{N} p\left(\nu \mid Z_{t}^{(i)}\right)
$$

Here the conditional posterior $p\left(\nu \mid Z_{t}^{(i)}\right)$ will include the dependence on $\sigma^{2}$ for the iid- $t$ case and $\left(\alpha, \beta, \tau^{2}\right)$ and the latent volatilities $h^{t}=\left(h_{1}, \ldots, h_{t}\right)$ for the SV- $t$ case through the essential state vector.

The task of sequential Bayesian computation is then equivalent to a filtering problem for the essential state vector, drawing $\left\{Z_{t}^{(i)}\right\}_{i=1}^{N} \sim p\left(Z_{t} \mid y^{t}\right)$ sequentially from the set of posteriors. To this end, PL exploits the following sequential decomposition of Bayes' rule

$$
\begin{aligned}
p\left(Z_{t+1} \mid y^{t+1}\right) & =\int p\left(Z_{t+1} \mid Z_{t}, y_{t+1}\right) d \mathbb{P}\left(Z_{t} \mid y^{t+1}\right) \\
& \propto \int \underbrace{p\left(Z_{t+1} \mid Z_{t}, y_{t+1}\right)}_{\text {propagate }} \underbrace{p\left(y_{t+1} \mid Z_{t}\right)}_{\text {resample }} d \mathbb{P}\left(Z_{t} \mid y^{t}\right) .
\end{aligned}
$$

The distribution $d \mathbb{P}\left(Z_{t} \mid y^{t+1}\right) \propto p\left(y_{t+1} \mid Z_{t}\right) d \mathbb{P}\left(Z_{t} \mid y^{t}\right)$ is a 1-step smoothing distribution. Here $\mathbb{P}\left(Z_{t} \mid y^{t}\right)$ denotes the current distribution of the current state vector and in particle form corresponds to $\frac{1}{N} \sum_{i=1}^{N} \delta_{Z_{t}^{(i)}}$, with $\delta$ a Dirac measure.

Bayes rule above then gives us a prescription for constructing a sequential simulationbased algorithm: given $\mathbb{P}\left(Z_{t} \mid y^{t}\right)$, find the smoothed distribution $\mathbb{P}\left(Z_{t} \mid y^{t+1}\right)$ via resampling and then propagate forward using $p\left(Z_{t+1} \mid Z_{t}, y_{t+1}\right)$. This simply finds draws from 
the next filtering distribution $\mathbb{P}\left(Z_{t+1} \mid y^{t+1}\right)$. Parameter inference is then achieved offline using $p\left(\theta \mid Z_{t+1}\right)$.

From a sampling perspective, this leads to a very simple algorithm for updating particles $\left\{Z_{t}\right\}_{i=1}^{N}$ to $\left\{Z_{t+1}\right\}_{i=1}^{N}$ in 3 steps:

1. Resample: with replacement from a multinomial with weights proportional to the predictive distribution $p\left(y_{t+1} \mid Z_{t}^{(i)}\right)$ to obtain $\left\{Z_{t}^{\zeta(i)}\right\}_{i=1}^{N}$;

2. Propagate: with $Z_{t+1}^{(i)} \sim p\left(Z_{t+1} \mid Z_{t}^{\zeta(i)}, y_{t+1}\right)$ to obtain $\left\{Z_{t+1}^{(i)}\right\}_{i=1}^{N}$;

3. Learning: $\nu$ from $p\left(\nu \mid Z_{t+1}\right)$.

The ingredients of particle learning are the essential state vector $Z_{t}$, a predictive probability rule $p\left(y_{t+1} \mid Z_{t}^{(i)}\right)$ for resampling $\zeta(i)$ and a propagation rule to update particles: $Z_{t}^{\zeta(i)} \rightarrow Z_{t+1}^{(i)}$. The essential state vector will include the necessary conditional sufficient statistics for parameter learning given a model specification.

\subsection{PL for the iid- $t$ case}

First, we consider the normal location-scale model of Section 2.1 with $\mu=0$ for simplicity. The model corresponds to a data augmentation scheme $\left(y_{t} \mid \sigma^{2}, \lambda_{t}\right) \sim$ $N\left(0, \sigma^{2} \lambda_{t}\right)$ with $\left(\lambda_{t} \mid \nu\right) \sim I G(\nu / 2, \nu / 2)$. To complete the model we assume priors of the form $\sigma^{2} \sim I G\left(n_{0} / 2, n_{0} \sigma_{0}^{2} / 2\right)$ and Jeffreys prior $p(\nu)$ for $\nu$ (equation 1 ).

Now, the key to our approach is the use of an essential state vector $Z_{t}$. The algorithm requires the following distributions: $p\left(y_{t+1} \mid Z_{t}\right), p\left(\nu, \sigma^{2} \mid Z_{t}\right)$ and $p\left(\lambda_{t} \mid \sigma^{2}, \nu, y_{t}\right)$. Bayes rule yields

$$
p\left(\nu \mid \lambda^{t}\right) \equiv p\left(\nu \mid S_{1, t}, S_{2, t}\right) \propto p(\nu)\left(\frac{\left(\frac{\nu}{2}\right)^{\frac{\nu}{2}}}{\Gamma\left(\frac{\nu}{2}\right)}\right)^{t} S_{1, t}^{-(\nu / 2+1)} \exp \left\{-\nu S_{2, t} / 2\right\}
$$


and

$$
p\left(\sigma^{2} \mid y^{t}, \lambda^{t}\right) \equiv p\left(\sigma^{2} \mid S_{3, t}, S_{4, t}\right) \sim I G\left(S_{3, t} / 2, S_{4, t} / 2\right)
$$

with recursive updates for the parameter sufficient statistics

$$
\begin{aligned}
& S_{t 1}=S_{t-1,1} \lambda_{t} \text { and } S_{t 2}=S_{t-1,2}+1 / \lambda_{t} \\
& S_{t 3}=S_{t-1,3}+1 \text { and } S_{t 4}=S_{t-1,4}+y_{t}^{2} / \lambda_{t}
\end{aligned}
$$

with initial values $S_{01}=1, S_{02}=0, S_{03}=n_{0}$ and $S_{04}=n_{0} \sigma_{0}^{2}$.

Additionally, the predictive distribution for resampling and the latent state conditional posterior for propagation are directly available as

$$
\begin{aligned}
p\left(y_{t+1} \mid \lambda_{t+1}, S_{t}\right) & \sim t_{S_{t 3}+2}\left(0, \frac{S_{t 4}}{S_{t 3}+2} \lambda_{t+1}\right) \\
p\left(\lambda_{t} \mid \mu, \sigma^{2}, \nu, y_{t}\right) & \sim I G\left(\frac{\nu+1}{2}, \frac{\nu+y_{t}^{2} / \sigma^{2}}{2}\right) .
\end{aligned}
$$

Therefore we use an essential state vector given by $Z_{t}=\left(\lambda_{t+1}, S_{t}\right)$. We are now ready to outline the steps of the PL scheme (see Panel A).

Although direct comparison with MCMC (Verdinelli and Wasserman, 1991) is not the focus of this paper, we observe that MCMC is inherently a non-sequential procedure. MCMC provides the full joint distribution $p\left(h^{T}, \theta, \nu \mid y^{T}\right)$ including smoothing of the initial volatility states particle learning only computes $p\left(h_{T}, \theta \mid y^{T}\right)$ - the distribution of the final state $h_{T}$ and parameters $\theta$. Another difference is in the assessment of MC errors. MCMC generates a dependent sequence of draws, PL has standard $\sqrt{N}$ MC bounds, but can suffer from accumulation of MC error for larger T. MCMC for learning fat-tails $\nu$ can exhibit low conductance (Eraker, Jacquier and Polson, 1998), having difficulty escaping lower values of $\nu$ in the chain, and can lead to poor 
convergence.

\subsection{PL for the SV- $t$ case}

Particle learning for the SV-t model is similar to the iid- $t$ model despite being somewhat more elaborated with the latent state now being the scale mixture $\lambda_{t}$ as well as the $\log$-volatilities $h_{t}$. In addition, there are three parameters $\left(\alpha, \beta, \tau^{2}\right)$ driving the log-volatility dynamic behavior, as opposed to $\sigma^{2}$ in the iid- $t$ model.

Static parameters. Let us first deal with $\theta=\left(\alpha, \beta, \tau^{2}\right)$ the vector of fixed parameters driving the log-volatility equation (see Section 2.2). Conditional on the latent volatilities $h^{t}=\left(h_{1}, \ldots, h_{t}\right)$, sampling $\theta$ is rather straightforward since it is based on the conjugate Bayesian analysis of the normal linear regression with $x_{t}^{\prime}=\left(1, h_{t-1}\right)$ (Gamerman and Lopes, 2006, Chapter 2), i.e. $\left(\alpha, \beta \mid \tau^{2}\right) \sim N\left(b_{t}, \tau^{2} B_{t}\right)$ and $\tau^{2} \sim I G\left(c_{t}, d_{t}\right)$. The parameter sufficient statistics are $S_{t}^{\theta}=\left(b_{t}, B_{t}, c_{t}, d_{t}\right)$ and they can determined recursively as

$$
\begin{aligned}
B_{t}^{-1} b_{t} & =B_{t-1}^{-1} b_{t-1}+h_{t} x_{t} \\
B_{t}^{-1} & =B_{t-1}^{-1}+x_{t} x_{t}^{\prime} \\
c_{t} & =c_{t-1}+1 / 2 \\
d_{t} & =d_{t-1}+\left(h_{t}-b_{t}^{\prime} x_{t}\right) h_{t} / 2+\left(b_{t-1}-b_{t}\right)^{\prime} B_{t-1}^{-1} b_{t-1} / 2 .
\end{aligned}
$$

Resampling step. To sequentially resample the log-volatility $h_{t}$ and propagate a new volatility state $h_{t+1}$, we use the Kim, Shephard and Chib (1998) strategy of approximating the distribution of $\log \tilde{y}_{t}^{2}$, where $\tilde{y}_{t}^{2}=y_{t}^{2} / \lambda_{t}$, by a carefully tuned seven- 
component mixture of normals ${ }^{2}$. Then, a standard data augmentation argument allows the mixture of normals to be conditionally transformed in individual normals, i.e. $\left(\varepsilon_{t} \mid k_{t}\right) \sim N\left(\mu_{k_{t}}, v_{k_{t}}^{2}\right)$, such that $k_{t} \sim \operatorname{Mult}(\pi)$. Conditionally on $k^{t}$, the SV-t model for $z_{k_{t}}=\log y_{t}^{2}-\log \lambda_{t}-\mu_{k_{t}}$ can be rewritten as a standard first order dynamic linear model, i.e.

$$
\begin{aligned}
\left(z_{k_{t}} \mid h_{t}, \lambda_{t}, k_{t}\right) & \sim N\left(h_{t}, v_{k_{t}}^{2}\right) \\
\left(h_{t} \mid h_{t-1}, \theta\right) & \sim N\left(\alpha+\beta h_{t-1}, \tau^{2}\right),
\end{aligned}
$$

with conditional state sufficient statistics $S_{t}^{h}=\left(m_{t}, C_{t}\right)$ given by the standard Kalman recursions (West and Harrison 1997). More explicitly, the conditional posterior $\left(h_{t} \mid S_{t}^{h}, \theta\right) \sim N\left(m_{t}, C_{t}\right)$ with moments given by

$$
m_{t}=\left(1-A_{t}\right) a_{t}+A_{t} z_{k_{t}} \quad \text { and } \quad C_{t}=\left(1-A_{t}\right) R_{t}
$$

where $a_{t}=\left(\alpha+\beta m_{t-1}\right), A_{t}=R_{t} / Q_{t}, R_{t}=\beta^{2} C_{t-1}+\tau^{2}$ and $Q_{t}=R_{t}+v_{k_{t}}^{2}$.

Essential state vector. We will take advantage of the above Kalman recursions in the resampling step. We use an essential state vector of the form

$$
Z_{t}=\left(\lambda_{t+1}, S_{t}^{\theta}, S_{t}^{\nu}, S_{t}^{h}\right)
$$

The subset $\left(S_{t}^{\theta}, S_{t}^{\nu}\right)$ of $Z_{t}$ is essentially the set $\left(S_{t 1}, \ldots, S_{t 4}\right)$ derived from the iid- $t$ model.

\footnotetext{
${ }^{2}$ More precisely, $\log \tilde{y}_{t}^{2}=h_{t}+\varepsilon_{t}$, where $\varepsilon_{t}=\log \epsilon_{t}^{2}$ follows a $\log \chi_{1}^{2}$ distribution, a parameter-free left skewed distribution with mean -1.27 and variance 4.94 . They show that the $\log \chi_{1}^{2}$ can be well approximated by $\sum_{j=1}^{7} \pi_{j} N\left(\mu_{j}, v_{j}^{2}\right)$, where $\pi=(0.0073,0.1056,0.00002,0.044,0.34,0.2457,0.2575)$, $\mu=(-11.4,-5.24,-9.84,1.51,-0.65,0.53,-2.36)$ and $v^{2}=(5.8,2.61,5.18,0.17,0.64,0.34,1.26)$.
} 
There are many efficiencies to be gained with this approach over traditional SMC approaches. For example, we only need to sample $h_{t-1}$ and $h_{t}$ (step 2) in order to propagate $S_{t}^{\theta}$ and sample $\theta$ (step 4). In other words, PL does not necessarily need to keep track of the log-volatilities. For instance, point-wise evaluations of $p\left(h_{t} \mid y^{t}\right)$ can be approximated by the Monte Carlo average of the Kalman filter densities, i.e. $p^{N}\left(h_{t} \mid y^{t}\right)=\frac{1}{N} \sum_{i=1}^{N} p\left(h_{t} ; m_{t}^{(i)}, C_{t}^{(i)}\right)$.

For estimation of the fat-tails, we can use a Rao-Blackwellised density estimate. For example in the SV-t case, in order to reduce Monte Carlo error, we use an estimate of the form

$$
p\left(\nu \mid y^{t}\right)=\mathbb{E}\left\{p\left(\nu \mid \lambda_{t}, h_{t}, y^{t}\right)\right\} \approx \frac{1}{N} \sum_{i=1}^{N} p\left(\nu \mid\left(\lambda^{t}, h^{t}\right)^{(i)}, y^{t}\right)
$$

where $\left\{\left(\lambda^{t}, h^{t}\right)^{(i)}\right\}_{i=1}^{N}$ are draws from $p\left(\lambda^{t}, h^{t} \mid y^{t}\right)$. This leads to efficiency gains as the conditional $p\left(\nu \mid \lambda^{t}, h^{t}, y^{t}\right)$ and conditional mean $\mathbb{E}\left(\nu \mid \lambda^{t}, h^{t}, y^{t}\right)$ are known in closed form. We are now ready to outline the steps of the PL scheme for the SV-t model (see Panel B).

\section{Model assessment with a sequential Bayes factor}

Sequential model determination is performed using a Bayes factor $\mathcal{B F}_{T}$ (Jeffreys, 1961, West, 1984). This naturally extends to a sequential version for an infinite sequence of (dependent) data we will still identify the "true" model. A probabilistic approach for determining how quickly you can learn the tail of the error distribution 
is to use the recursion

$$
\mathcal{B F}_{T+1}=\frac{p\left(y_{T+1} \mid y_{1}, \ldots, y_{T}\right)}{q\left(y_{T+1} \mid y_{1}, \ldots, y_{T}\right)} \mathcal{B F}_{T}
$$

Blackwell and Dubins (1962) provide a general discussion of the merging of opinions under Bayesian learning. They show that for any two models $p\left(y_{1}, \ldots, y_{T}\right)$ and $q\left(y_{1}, \ldots, y_{T}\right)$ that are absolutely continuous with respect to each other, opinions that merge in the following sense. First, $\mathcal{B F}_{T}$ is a martingale, $\mathcal{F}_{T}$-measurable and under $Q$,

$$
\mathbb{E}_{Q}\left(\frac{p\left(y_{T+1} \mid y_{1}, \ldots, y_{T}\right)}{q\left(y_{T+1} \mid y_{1}, \ldots, y_{T}\right)} \mid \mathcal{F}_{T}\right)=1 \text { so that } \mathbb{E}\left(\mathcal{B F}_{T+1} \mid \mathcal{F}_{T}\right)=\mathcal{B F}_{T}
$$

By the martingale convergence theorem, $\mathcal{B F}_{\infty}=\lim _{T \rightarrow \infty} \mathcal{B F}_{T}$ exists almost surely under $Q$ and in fact $\mathcal{B F}_{\infty}=0$ a.s. $Q$. Put simply, the sequential Bayes factor will correctly identify the 'true' model $Q$ under quite general data sequences include the SV- $t$ model we consider here in detail. Furthermore, by the Shannon-McMillanBreiman theorem (see, for example, Cover and Thomas, 2006) we can analyse the rate of learning via the quantity

$$
\lim _{T \rightarrow \infty} \frac{1}{T} \ln q\left(y_{1}, \ldots, y_{T}\right) \rightarrow H \text { a.s. } Q,
$$

where $H$ is the entropy rate defined by $H=\lim _{T \rightarrow \infty} \mathbb{E}_{Q}\left(-\ln p\left(y_{T+1} \mid y_{1}, \ldots, y_{T}\right)\right)<0$. Hence as $H \in[-\infty, 0)$ we have that $\mathcal{B F}_{\infty}=0$. A similar result for the marginal likelihood ratio

$$
\lim _{T \rightarrow \infty} \frac{1}{T} \ln \frac{p\left(y_{1}, \ldots, y_{T}\right)}{q\left(y_{1}, \ldots, y_{T}\right)} \rightarrow \lim _{k \rightarrow \infty} \mathbb{E}_{Q}\left(\ln \frac{p\left(y_{k+1} \mid y_{k}, \ldots, y_{1}\right)}{q\left(y_{k+1} \mid y_{k}, \ldots, y_{1}\right)}\right)<0 \text { a.s. } Q \text {. }
$$


We will use this in the next subsection.

Bayes factors have a number of attractive features as they can be converted into posterior model probabilities when the model set is exhaustive. Lopes and Tobias (2010) provide a recent survey including computational strategies based on the Savage-Dickey density ratio. These results are only asymptotic and with a finite amount of data it helps to analyze the rate of learning using a Kullback-Leibler metric.

\subsection{Discriminating a $t_{4}$ from a Gaussian}

We can use these theoretical insights (see also Edwards, Lindman and Savage, 1963, Lindley, 1956) to address the question a priori of "how long a time series one would have to observe to have strong evidence of a $t_{4}$ versus a Gaussian"? Jeffreys observed that you need data sequences of length $T=500$ to be able to discriminate the tails of an underlying probability distribution. We now formalize this argument using our sequential Bayes factor. One is motivated to define a priori the "expected" log-Bayes factor for a given data length, $\overline{\mathcal{B F}}_{T}$, under the Gaussian model

$$
\frac{1}{T} \ln \overline{\mathcal{B F}}_{T}=\mathbb{E}_{t_{\infty}} \ln \frac{t_{\nu}}{t_{\infty}}=K L\left(t_{\nu}, t_{\infty}\right)
$$

under the Gaussian $t_{\infty}$-model where $K L$ denotes Kullback-Leibler divergence. Then, a priori, if we are given a level of Bayes factor discrimination $\overline{\mathcal{B F}}_{T}$ we then have to observe on average $T^{\star}$ observation to be able to discriminate the two models where

$$
T^{\star}=\frac{1}{K L\left(t_{\nu}, t_{\infty}\right)} \ln \overline{\mathcal{B F}}_{T}
$$


This measure is asymmetric, as if the data is generated by a $t_{\nu}$ distribution, the constant changes to $K L\left(t_{\infty}, t_{\nu}\right)$.

To illustrate the magnitudes of these effects, if we take $\nu=3$ and $\mathcal{B F}=10$ (strong evidence), for example, this argument would suggest that on average $T=150$ observations from a standard normal are needed to strongly reject the $t_{3}$ model, and on average $T=20$ observations from the $t_{3}$ to strongly reject the standard normal distribution. This is borne out in our empirical study. Figure 1 plots the first factor in the above expression namely the Kullback-Leibler divergence between the $t_{\nu}$-family and the Gaussian.

This also confirms the analysis in Gramacy and Pantaleo (2010). In a multivariate regression setting, they perform a Monte Carlo experiment where $T$ and $\nu$ varied with $T \in\{30,75,100,200,500,1000\}$ and $\nu \in\{3,5,7,10, \infty\}$. They observed the frequency of time the $\mathcal{B F}$ indicated strong preference $(\mathcal{B F}>10)$ for a model. Under normal errors, $\nu=3$ could be determined with high accuracy for $T \leq 200, \nu=5$ took $T \leq 1000$ and for $10 \leq \nu<\infty$ very large samples would be required to discriminate the tails with any degree of posterior accuracy. Of course, for a given dataset, the Bayes factor might provide strong evidence even for small samples. The Jeffreys prior then has the nice property (by definition of the inverse of the Fisher information matrix) of down-weighting these regions of the parameter space where it is hard to learn the parameters.

It is also interesting to address the asymptotic behavior of the fat-tailed posterior distribution when the true model is not in the set of models under consideration. Berk $(1966,1970)$ assumes that the data generating process comes from $y_{t} \sim q(y)-$ a model outside our current consideration. Given our fat-tailed model $p(y \mid \theta, \nu)$, Berk shows that under mild regularity conditions that the posterior distribution $p(\theta, \nu \mid y)$ will 
asymptotically concentrate with probability one on the subset of parameter values where the Kullback-Leibler divergence between $p(y \mid \theta, \nu)$ and $q(y)$ is minimized or equivalently $\int \log p(y \mid \theta, \nu) q(y) d y$ is maximized.

\section{Empirical results}

We now illustrate our methodology for iid SV-Student's $t$ error distributions (see Sections 2.1 and 2.2 for the specifications). The iid- $t$ model illustration will serve the additional and important purpose of showing that the uniform prior is not necessarily always a harmless prior. The SV-t model will be estimated sequentially on the British pound/US dollar daily exchange rate series and daily returns on the S\&P500 from a period in 2007-2010 that includes the credit crisis. Resulting inferences will be compared with MCMC at the end of the sample.

\subsection{The iid- $t$ model}

To illustrate the efficiency of our approach, we simulate a sample of size $T=200$ from a Student's $t_{4}$ distribution, centered at zero and unit scale, i.e. $\sigma^{2}=1$. Figures 2 and 3 show the joint posterior distributions of $p\left(\sigma^{2}, \nu \mid y^{t}\right)$ for $t=50,100,150$ and 200 under, respectively, the uniform prior and the Jeffreys prior of Fonseca et al. (2008). As the model implies that $\operatorname{Var}\left(y_{t}\right)=\sigma^{2} \nu /(\nu-2)$ one should not be too surprised that there is a posterior correlation between $\sigma^{2}$ and $\nu$ for small values of $\nu$.

It is clear that the posterior provides fairly accurate sequential estimates for the joint as well as the marginal distributions (the exact posterior probabilities are com-

puted on a fine bivariate grid). On the one hand, the Jeffreys prior, as anticipated, penalizes larger values of $\nu$ with the penalization slightly decreasing as the sample 
size increases. On the other hand, the uniform prior is impartial with respect to the number of degrees of freedom, so any information regarding $\nu$ comes exclusively from the likelihood which, in turn, is fairly uninformative about $\nu$ for $t=50,100$ and 150 . Even when $t=200$, there is still no negligible mass for values $\nu>10$. Figure 4 shows that PL estimates are still accurate when $n=1000$. It also shows that the marginal posterior of $\nu$ is highly concentrated around the true value for $t>500$, as theoretically predictive in Section 4.1 and Figure 1.

The undesirable bias of the not-so-harmless uniform prior is highlighted in the Monte Carlo exercise summarized by Figures 5 and 6 . The posterior means, medians and modes of $\nu$ based on $p\left(\nu \mid y^{t}\right), t=30,50,100,300,400$ and 500 are compared across $R=50$ samples. As it can be seen, the bias of the uniform prior is striking for samples

of size up to $T=100$, when compared to those of the Jeffreys prior. For samples of size $T=400$ and $T=500$ the bias is much smaller, but a closer look reveals its presence. For example, the 25th percentiles of the mean, median and mode box-plots when $T=500$ are all above the true value $\nu=4$ for the uniform prior.

\subsection{The SV- $t$ model}

We now revisit the well-known British Pound versus US Dollar exchange rate data of Jacquier et al. (2004). The data consists of $T=937$ daily rates form October 1st, 1981 to June, 28th 1985. For illustration purposes, we simulated data with exactly the same length from a SV- $t_{4}$ model with parameters $\left(\nu, \alpha, \beta, \tau^{2}\right)=(4,-0.202,0.980,0.018)$ and initial value $h_{0}=-8.053$. Both simulated and real data sets are presented in Figure 7.

The prior distribution of $\nu$ is given by the discretized version of Fonseca et al.'s (2008) Jeffreys prior, similar to the approach taken in Section 5.1 (see equation 1). 
The vector log-volatility parameters $\left(\alpha, \beta, \tau^{2}\right)$ are independent, a priori, of $\nu$ and its prior distribution is given by $(\alpha, \beta) \mid \tau^{2} \sim N\left(b_{0}, \tau^{2} B_{0}\right)$ and $\tau^{2} \sim I G\left(\eta_{0} / 2, \eta_{0} \tau_{0}^{2} / 2\right)$, while the posterior for the log-volatility at time $t=0$ is given by $h_{0} \sim N\left(m_{0}, C_{0}\right)$. The hyper-parameters are set at the values $m_{0}=\log y_{1}^{2}, C_{0}=1.0, b_{0}=(-0.002,0.97)$, $B_{0}=\operatorname{diag}(1.0,0.01), c_{0}=5.0$ and $d_{0}=0.1125$.

Posterior inference is based on PL with $N=10,000$ particles. Figures 8 presents $2.5 \mathrm{th}, 50 \mathrm{th}$ and 97.5 th percentiles of the sequential marginal distributions of $\alpha, \beta$, $\tau^{2}$ and $\nu$ for both simulated and real data sets. For the simulated data, the posterior distribution of $\nu$ concentrates around the true value $\nu=4$ after about 350 observations. For the real data, $\nu$ is highly concentrated with around ten degrees of freedom at the end of the sample; however the right tail of the distribution, i.e. large degrees of freedom, is fairly long for most of the sample. Another interesting fact is that both normal and Student's $t$ model learn about $\alpha$ and $\beta$ in a similar manner, while the same cannot be said for the volatility of the log-volatility parameter, $\tau^{2}$. This is perhaps not surprising as the normal model overestimates the volatility of log-volatility to accommodate the fact that daily rates violate the plain normality assumption. The same behavior is present in our simulated data exercise. In fact, the posterior distribution for the log-volatilities, $p\left(h_{t} \mid y^{t}\right)$, for the simulated data based on the normal model has larger uncertainty than for the $t_{\nu}$ model (Figure not shown here). Finally, at the end of the sample we can calculate the marginal posterior on the tail-thickness $p\left(\nu \mid y^{T}\right)$, our sequential particle approach agrees with the MCMC analysis of Jacquier et al. (2004). This suggests that the MC accumulation error inherent in our particle algorithm is small for these types of data length and models. 


\subsubsection{S\&P500: Credit Crisis 2008-2009}

To study the effect of the credit crisis on stock returns we revisit daily S\&P500 returns previously studied, amongst many others, by Abanto-Valle et al. (2010) and Lopes and Polson (2010b). The former paper estimates SV models with errors in the class of symmetric scale mixtures of normal distributions and also base their illustration on the S\&P500 index from January 1999 to September 2008, therefore missing most of the credit crunch crisis and its aftermath. We concentrate our analysis on the period starting on January 3rd 2007 and ending on October 14th 2010 ( $T=954$ observations). We sequentially fit the normal model to this data set as well as the $t_{\nu}$ model for $\nu \in\{5,10,50\}$. Figure 9 summarizes our findings. The three Student's $t$ models have higher predictive power than the normal model when measured in terms of $\log$-Bayes factors. This distinction is particularly strong when comparing the $t_{5}$ (or $t_{10}$ ) model with the normal model. Interestingly, the $t_{5}$ model becomes gradually closer to the normal model from July 2008 to July 2010, when again distances itself from normality.

Before the onset of the credit crisis in July 2008, the model with the largest Bayes factor (relative to a normal) and hence the largest posterior model probability (under a uniform prior on $\nu$ ) is the $t_{5}$-distribution. This is maybe not surprising as the previous time period consisted of little stochastic volatility and the occasional outlying return - which is nicely accomodated by a $t_{5}$ error distribution, in the spirit of Jeffreys initial observation about "real" data. The interesing aspects of Bayesian learning occur in the period of the crisis from July 2008 to March 2009. One immediately sees a dramatic increase the stochastic volatility component of the model and the clustering of a high period of volatility. In and of itself this is sufficient to "explain" the extreme moves in the market. Corresponding, in terms of online estimation of 
the fat-tails the Bayes factor quickly moves to favor the model with light tails, here the $t_{10}$-distribution. Finally, as the crisis subsides, the volatility mean reverts and the returns again look like they exhibit some outlying behavior (relative to the level of volatility) and the sequential Bayes again starts to move to favor the fatter-tailed $t_{5}$-distribution.

\section{Discussion}

Estimating tail-thickness of the error distribution of an economic or financial time series is an important problem as estimates and forecasts are very sensitive to the tail behavior. Moreover, we would like an on-line estimation methodology that can adaptively learn the tail-thickness and provide parameter estimates that update as new data arrives. We model the error distribution as a $t_{\nu}$-distribution where $\nu \sim p(\nu)$ and we adopt a default Jeffreys prior on the tail-thickness parameter $\nu$. We show that this has a number of desirable properties when performing inference with a finite amount of data. We use the sequential Bayes factor to provide an on-line test of normality versus fat-tails and we derive its optimality properties asymptotically and in finite sample using a Kullback-Leibler metric. We illustrate these effects in the credit crisis of 2008-2009 with daily S\&P500 stock return data. Our analysis shows how quickly an agent can dynamically learn the tail of the error distribution whilst still accounting for parameter uncertainty and time-varying stochastic volatility.

Whilst MCMC is computationally slow for solving the online problem it does also provides the full smoothing distribution at the end of the sampler. This would require $O\left(N^{2}\right)$ particles in our approach (see Carvalho et al., 2010, for further discussion) and therefore if smoothed states are required we recommend filtering forward with parti- 
cles and smoothing with MCMC. Other estimation methods such as nested Laplace approximation (Smith, 2000) seem unable to identify the true error structure due to the multi-modalities present in the posterior and particle methods provide a natural alternative. Clearly there are a number of extensions of our approach for example to multivariate and dynamic panel data. 


\section{References}

Abanto-Valle, C.A., D. Bandyopadhyay, V.H. Lachos, I. Enriquez, 2010, Robust Bayesian analysis of heavy-tailed stochastic volatility models using scale mixtures of normal distributions. Computational Statistics and Data Analysis, 54, 2883-2898. Andrews, D.F. and C.L. Mallows, 1974, Scale mixtures of normal distributions. Journal of Royal Statistical Society, Series B, 36, 99-102.

Berk, R.H., 1966, Limiting behavior of posterior distributions when the model is incorrect. Annals of Mathematical Statistics, 37, 51-58.

Berk, R.H., 1970, Consistency a posteriori. Annals of Mathematical Statistics, 41, 894-906.

Blackwell, D. and Dubins, L., 1962, Merging of opinions with increasing information. Annals of Mathematical Statistics, 33, 882-886.

Carlin, B.P., N.G. Polson and D.S. Stoffer, 1992, A Monte Carlo approach to nonlinear and non-normal state space models. Journal of the American Statistical Association, 87, 493-500.

Carvalho, C.M., M.S. Johannes, H.F. Lopes and N.G. Polson ,2010, Particle learning and smoothing. Statistical Science, 25, 88-106.

Chen, R. and J. Liu, 2000, Mixture Kalman filters. Journal of Royal Statistical Society, Series B, 62, 493-508.

Chib, S., F. Nardari and N. Shephard, 2002, Markov chain Monte Carlo methods for stochastic volatility models. Journal of Econometrics, 108, 281-316.

Cover, T.M. and J.A. Thomas, 2006, Elements of Information Theory (2nd edition) Wiley, New York. 
Edgeworth, F.Y., 1888, On a new method of reducing observations relating to several quantities. Philosophical Magazine, 25, 184-191.

Edwards, W., H. Lindman and L.J. Savage ,1963, Bayesian statistical inference for psychological research Psychological Review, 70, 193-242.

Eraker, B., E. Jacquier and N.G. Polson, 1998, The pitfalls of MCMC algorithms. Technical Report, The University of Chicago Booth School of Business.

Fernandez, C. and M.F.J. Steel,1998, On Bayesian modeling of fat tails and skewness. Journal of the American Statistical Association, 93, 359-371.

Fonseca, T., M.A.R. Ferreira and H.S. Migon, 2008, Objective Bayesian analysis for the Student- $t$ regression model. Biometrika, 95, 325-333.

Frühwirth-Schnatter, S., 2006, Finite mixture and Markov switching models SpringerVerlag, New York.

Gamerman, D. and H.F. Lopes, 2006, Markov chain Monte Carlo: stochastic simulation for Bayesian inference Chapman \& Hall/CRC, Baton Rouge.

Geweke, J., 1993, Bayesian treatment of the independent Student- $t$ linear linear model. Journal of Applied Econometrics, 8, 19-40.

Gordon, N., D. Salmond and A.F.M. Smith, 1993, Novel approach to nonlinear/nonGaussian Bayesian state estimation. IEE Proceedings, F-140, 107-113.

Gordon, N. and A.F.M. Smith, 1993, Approximate non-Gaussian Bayesian estimation and modal consistency. Journal of Royal Statistical Society, Series B, 55, 913-918.

Gramacy, R. and E. Pantaleo, 2010, Shrinkage regression for multivariate inference with missing data, and an application to portfolio balancing. Bayesian Analysis, 5, $237-262$. 
Jacquier, E. and N.G. Polson, 2000, Discussion of "Time series analysis of nonGaussian observations". Journal of Royal Statistical Society, B, 62, 44-45.

Jacquier E., N.G. Polson and P.E. Rossi, 2004, Bayesian analysis of stochastic volatility with fat tails and leverage effect. Journal of Econometrics, 122, 185-212.

Jeffreys, H., 1961, Theory of probability Oxford University Press, New York.

Lindley, D.V., 1956, On a measure of the information provided by an experiment. Annals of Mathematical Statistics, 27, 986-1005.

Lopes, H.F., C.M. Carvalho, M.S. Johannes and N.G. Polson, 2010, Particle learning for sequential Bayesian computation (with discussion), in: J.M. Bernardo, M.J. Bayarri, J.O. Berger, A.P. Dawid, D. Heckerman, A.F.M. Smith and M. West, (Eds.), Bayesian statistics, Vol. 9. Oxford University Press, Oxford. (to appear)

Lopes, H.F. and N.G. Polson, 2010a, Bayesian inference for stochastic volatility modeling. In K. Böcker (Ed.), Re-Thinking risk measurement, management and reporting measurement uncertainty, Bayesian analysis and expert elicitation, Riskbooks, pp. $515-551$.

Lopes H.F. and N.G. Polson, 2010b, Extracting SP500 and NASDAQ volatility: The credit crisis of 2007-2008, in: A. O'Hagan and M. West, (Eds.), Handbook of applied Bayesian analysis, Oxford University Press, Oxford, pp. 319-342.

Lopes, H.F. and J. Tobias, 2010, Confronting prior convictions: On issues of prior and likelihood sensitivity in Bayesian analysis. Annual Review of Economics, Volume 3. (to appear)

McCausland, W., 2008, The Hessian method (highly efficient state smoothing, in a nutshell, Working Paper Series, no. 2008-03, University of Montreal, Department of Economics. 
Smith, A.F.M., 1983, Bayesian approaches to outliers and robustness. In J.P. Florens, M. Mouchart, J.P. Raoult, L. Simar and A.F.M. Smith, (Eds.), Specifying statistical models: from parametric to nonparametric, using Bayesian or non-Bayesian approaches, Springer-Verlag, New York, pp. 13-35.

Smith, J.Q., 2000, In discussion of "Time series analysis of non-Gaussian observations". Journal of Royal Statistical Society, B, 62, 29-20.

Verdinelli, I. and L. Wasserman, 1995, Computing Bayes factors by using a generalization of the Savage-Dickey density ratio. Journal of the American Statistical Association, 90, 614-618.

West, M., 1981, Robust sequential approximate Bayesian estimation. Journal of Royal Statistical Society, Series B, 43, 157-166.

West, M., 1984, Bayesian model monitoring. Journal of Royal Statistical Society, Series B, 48, 70-78. 


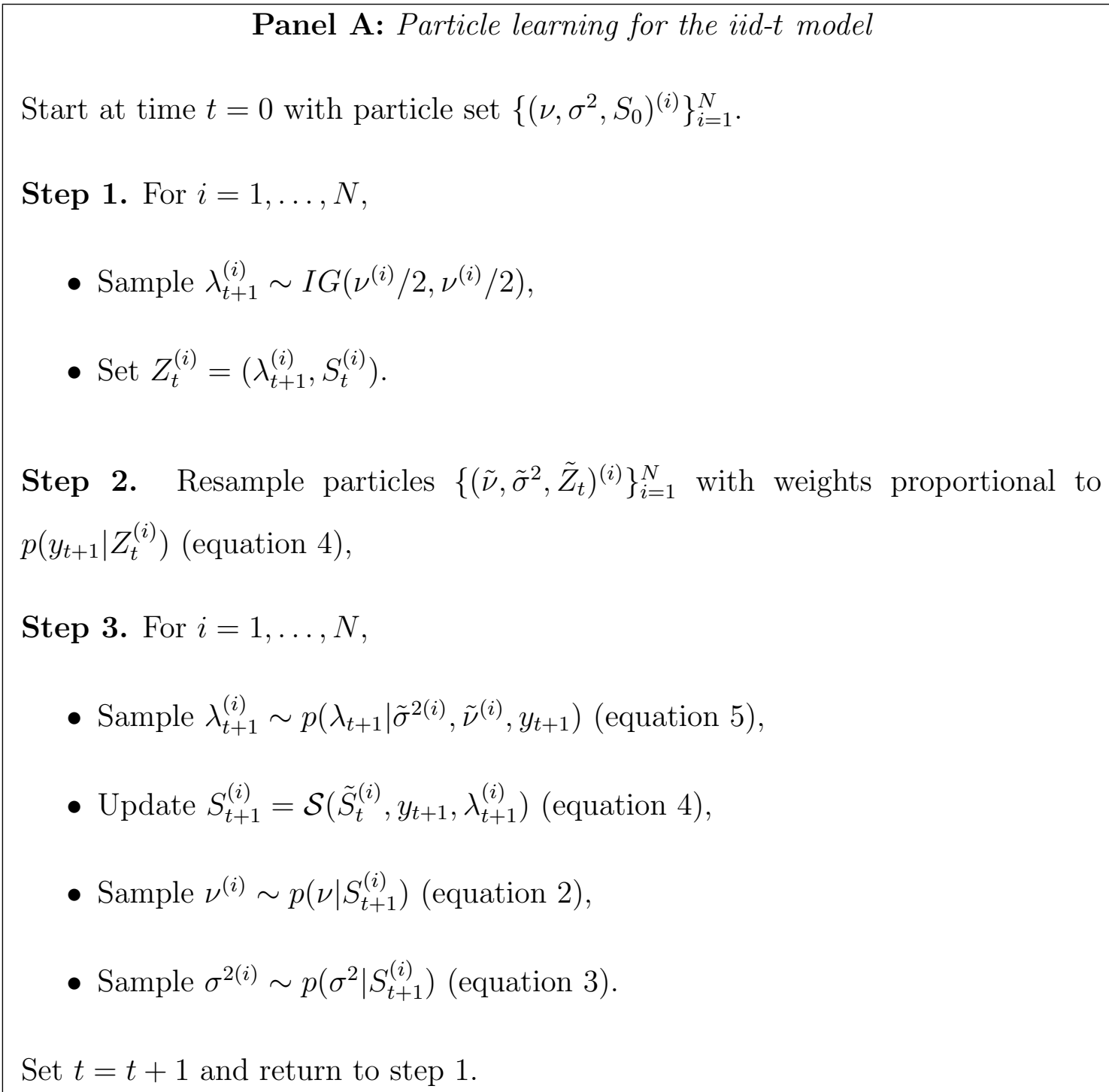




\section{Panel B: Particle learning for the SV-t model}

Step 0. Sample $\lambda_{t}^{(i)} \sim I G\left(\nu^{(i)} / 2, \nu^{(i)} / 2\right)$,

Step 1. Resample particles $\left\{\left(\tilde{S}_{t-1}^{\theta}, \tilde{S}_{t-1}^{h}, \tilde{\lambda}_{t}, \tilde{\theta}\right)\right\}_{i=1}^{N}$ with weights

$$
w_{t}^{(i)} \propto \sum_{k_{t}=1}^{7} \pi_{i} p_{N}\left(z_{k_{t}}^{(i)} ; a_{t}^{(i)}, Q_{t}^{(i)}\right)
$$

Step 2. Sample $\left(h_{t-1}, h_{t}\right)$ from $p\left(h_{t-1}, h_{t} \mid S_{t-1}^{h}, \lambda_{t}, \theta, y^{t}\right)$ :

Step 2.1. Sample $h_{t-1}$ from $\sum_{j=1}^{7} \pi_{j} f_{N}\left(h_{t-1} ; \hat{h}_{t-1, j}, V_{t-1, j}\right)$, where

$$
\begin{aligned}
& \hat{h}_{t-1, j}=V_{t-1, i}\left(m_{t-1} / C_{t-1}+z_{t i} \beta /\left(v_{i}^{2}+\tau^{2}\right)\right) \\
& V_{t-1, j}=1 /\left(1 / C_{t-1}+\beta^{2} /\left(v_{j}^{2}+\tau^{2}\right)\right)
\end{aligned}
$$

for $z_{t i}=\log y_{t}^{2}-\log \lambda_{t}-\mu_{i}-\alpha$,

Step 2.2. Sample $h_{t}$ from $=\sum_{j=1}^{7} \pi_{j} f_{N}\left(h_{t} ; \tilde{h}_{t j}, W_{t j}\right)$, where

$$
\begin{aligned}
\tilde{h}_{t i} & =W_{t i}\left(\tilde{z}_{t i} / v_{i}^{2}+\left(\alpha+\beta h_{t-1}\right) / \tau^{2}\right) \\
W_{t i} & =1 /\left(1 / v_{i}^{2}+1 / \tau^{2}\right)
\end{aligned}
$$

for $\tilde{z}_{t i}=\log y_{t}^{2}-\log \lambda_{t}-\mu_{i}$,

Step 3. Update $S_{t+1}^{\nu(i)}$ (equation 4); sample $\nu^{(i)} \sim p\left(\nu \mid S_{t+1}^{\nu(i)}\right)$ (equation 2),

Step 4. Update $S_{t}^{\theta(i)}$ (equation 6); sample $\theta \sim p\left(\theta \mid S_{t}^{\theta(i)}\right.$ ),

Step 5. Propagate $S_{t}^{h(i)}$ (equation 7). 

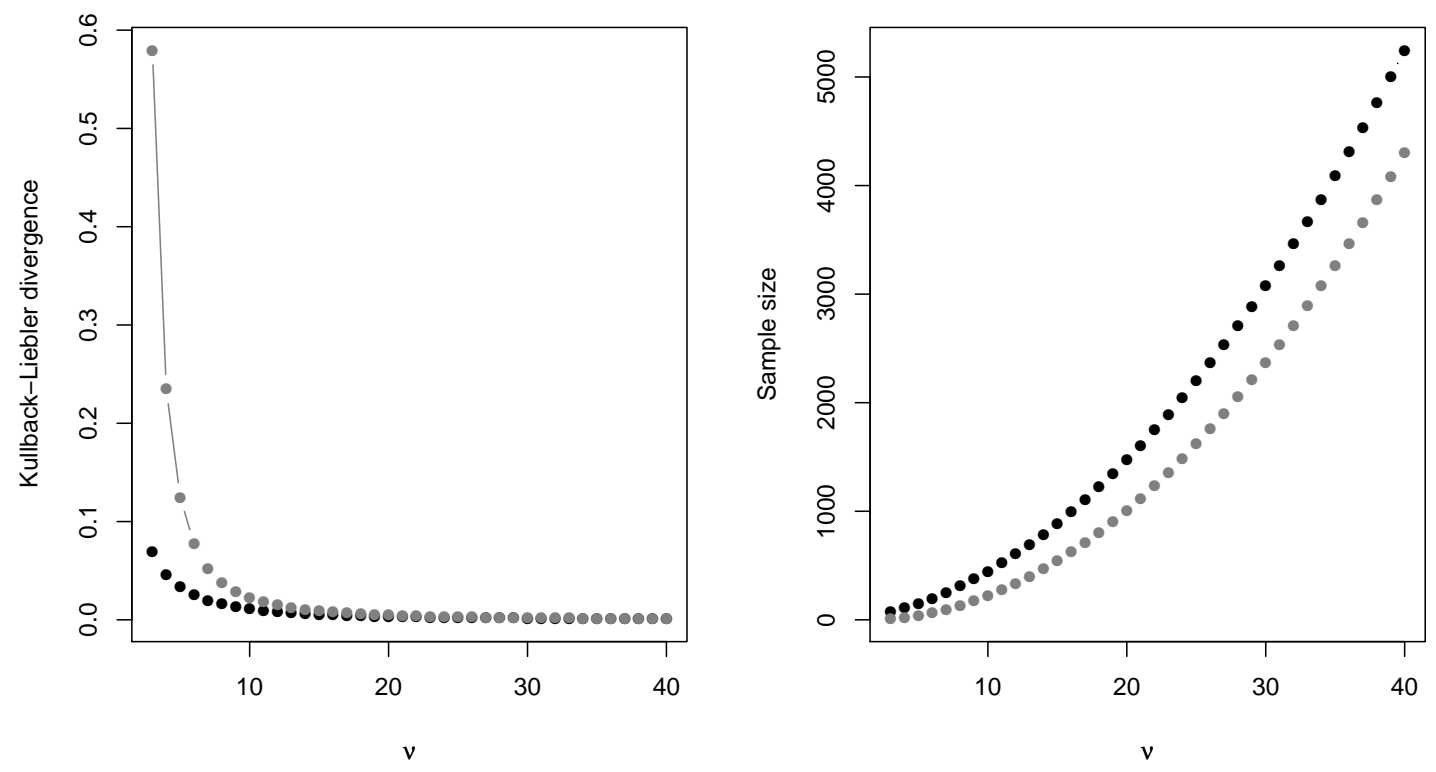

Figure 1: i.i.d. model. Discriminating a $t_{\nu}$ from a Gaussian. $K L\left(t_{\nu}, t_{\infty}\right)$ (black) and $K L\left(t_{\infty}, t_{\nu}\right)$ (grey). For $\nu=4,10,20$, theoretical sample sizes are $T^{*}=108,446,1473$ for strong evidence against normality and $T^{*}=22,220,1009$ for strong evidence against $t_{\nu}$. 

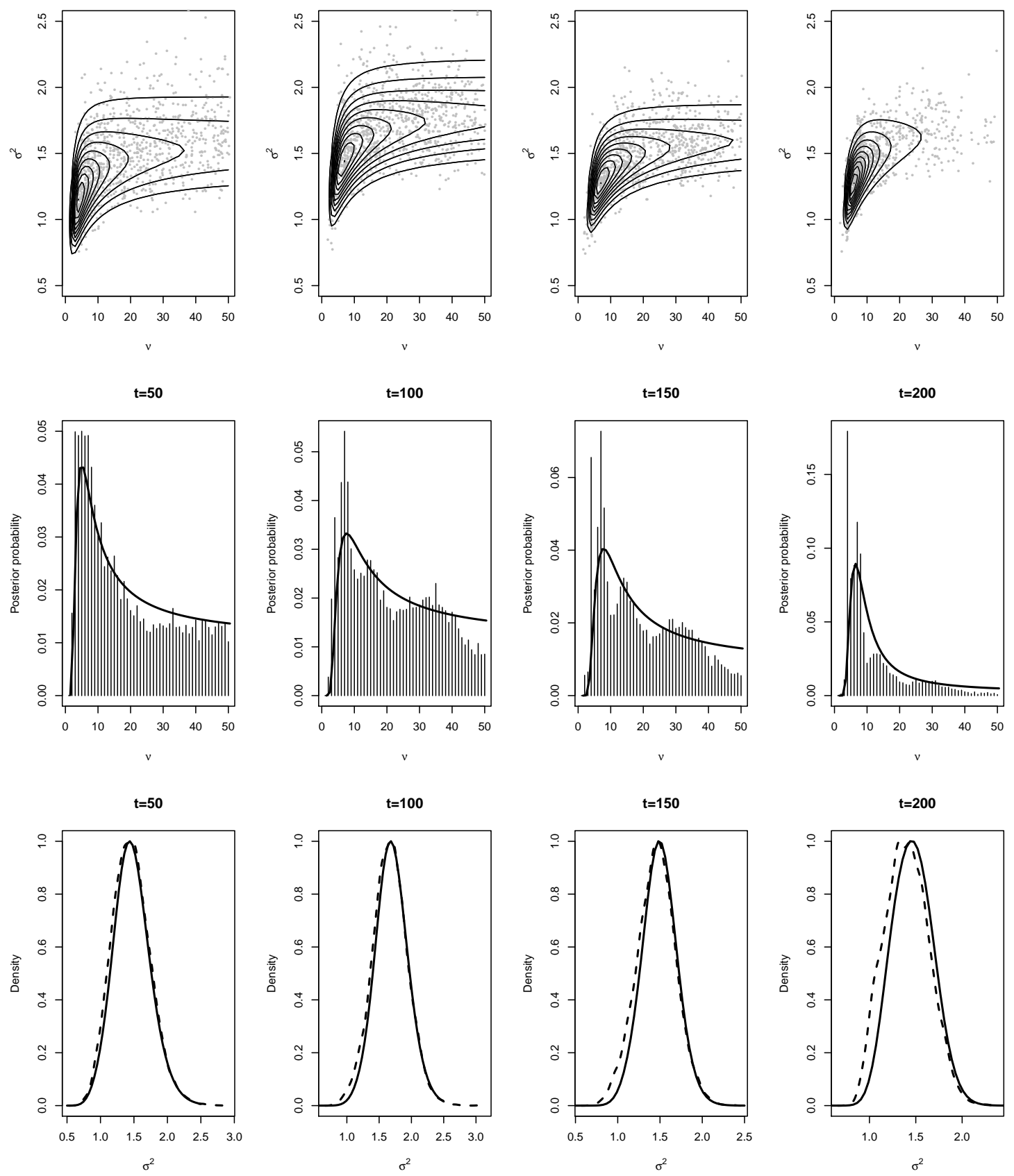

Figure 2: i.i.d. model. Sequential posterior inference for $\left(\sigma^{2}, \nu\right)$ based on PL for $T=200$ iid observations drawn from $t_{4}$ with uniform prior for $\nu$. PL is based on $N=10,000$ particles. 

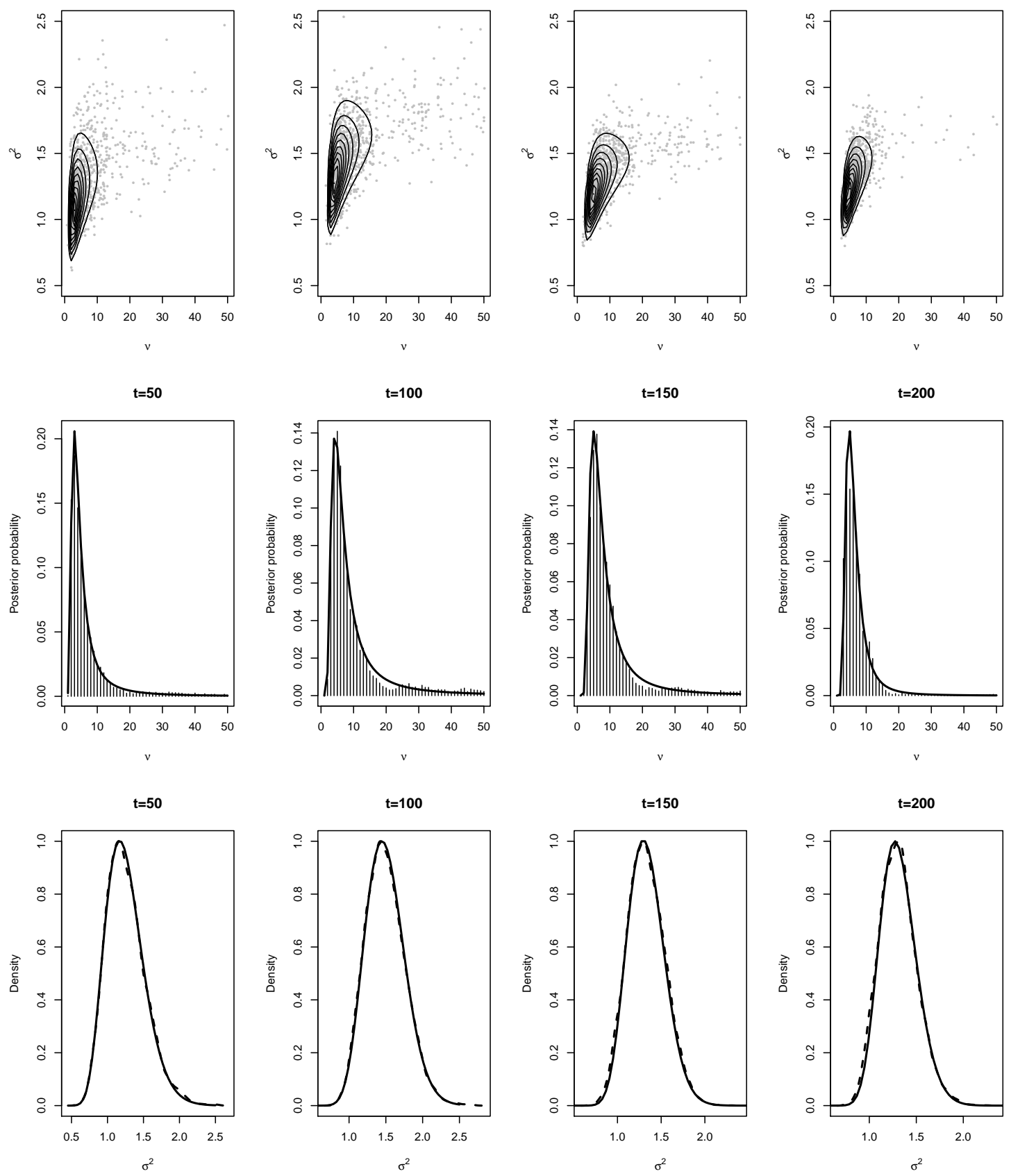

Figure 3: i.i.d. model. Sequential posterior inference for $\left(\sigma^{2}, \nu\right)$ based on PL for $T=200$ iid observations drawn from $t_{4}$ with Jeffreys prior for $\nu$. PL is based on $N=10,000$ particles. 

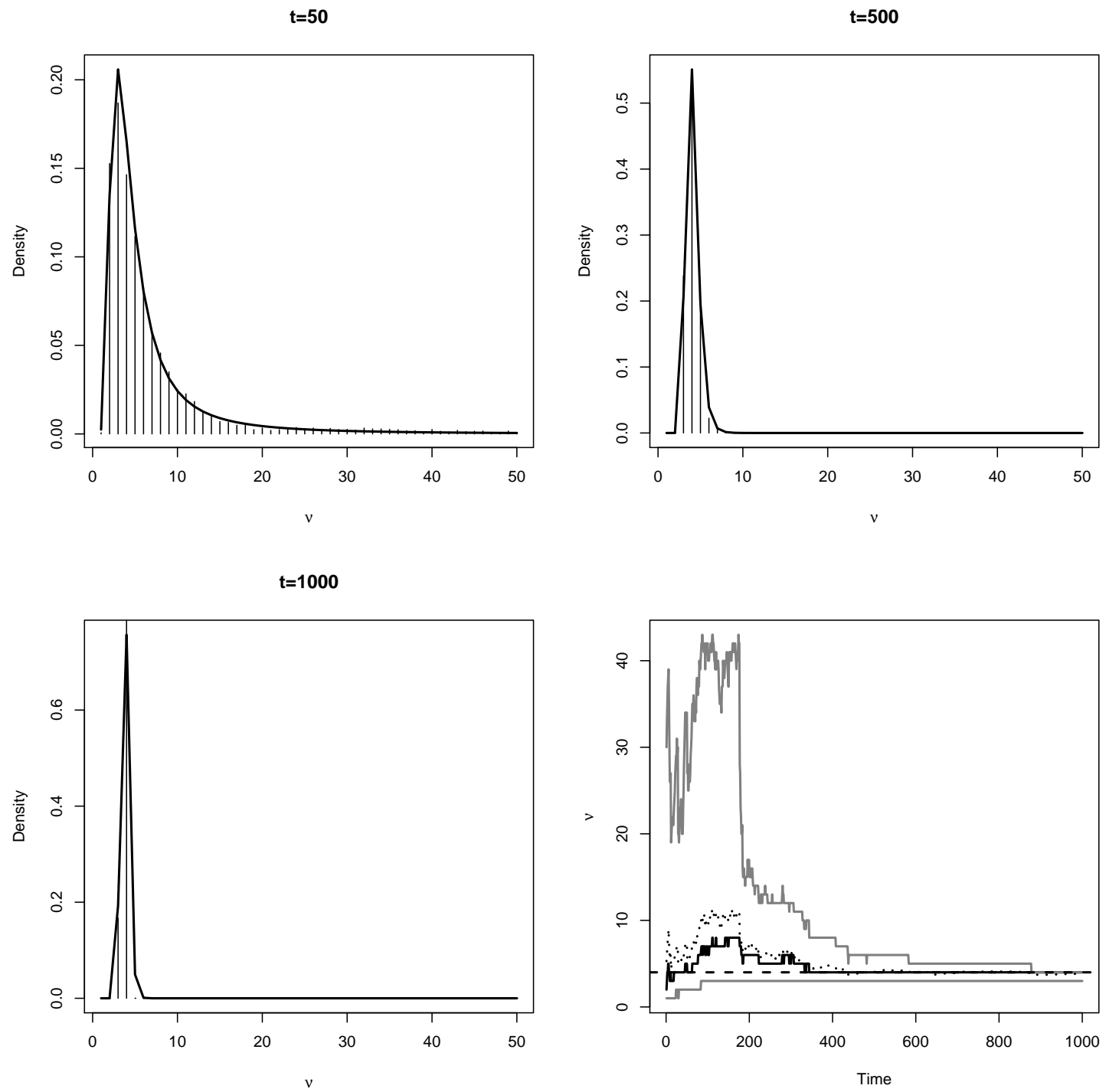

Figure 4: i.i.d. model. Sequential posterior inference for $\nu$ based on PL for $T=1000$ iid observations drawn from $t_{4}$ with Jeffreys prior for $\nu$. PL is based on $N=10,000$ particles. 

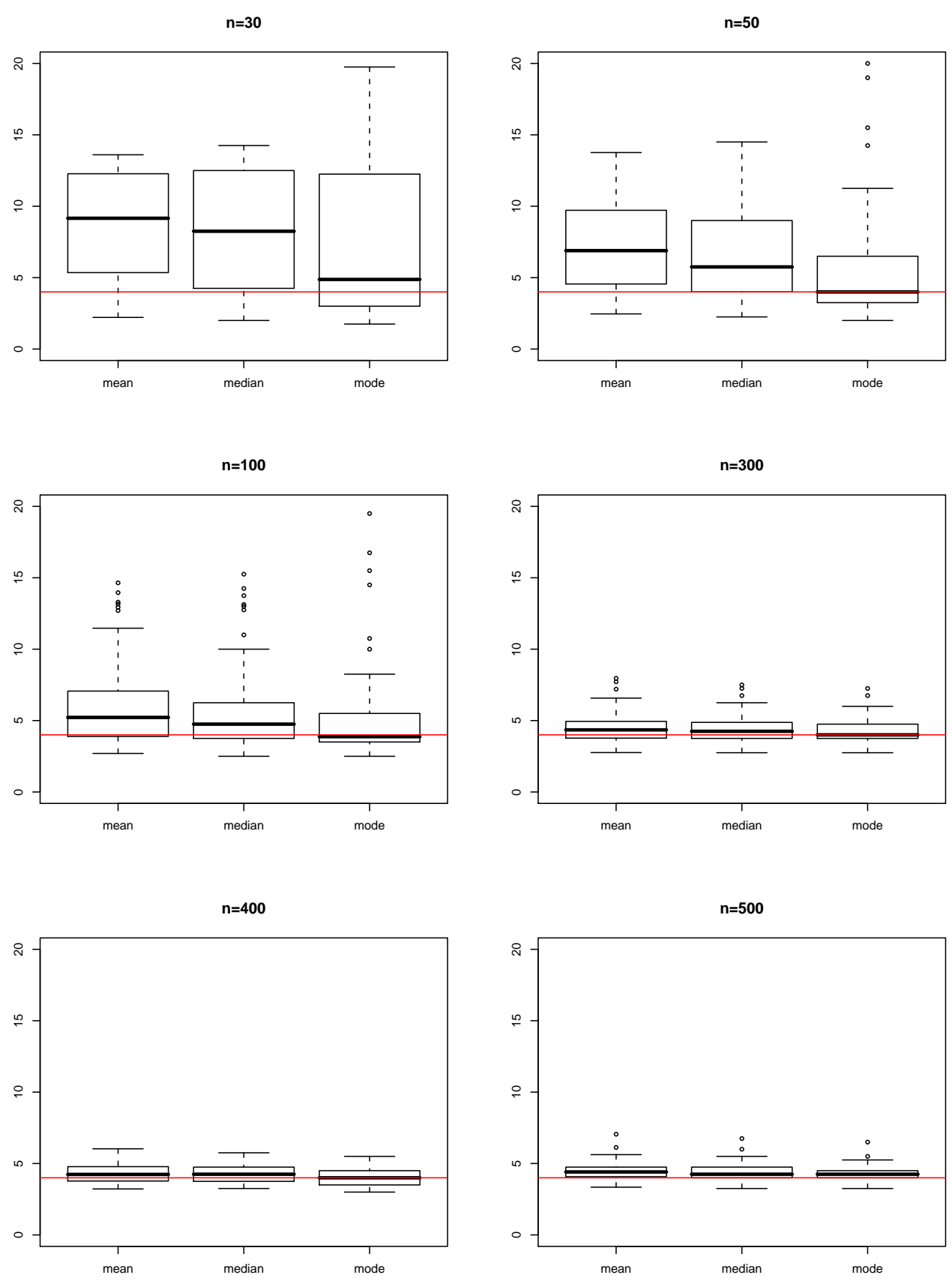

Figure 5: i.i.d. model. Posterior mean, median and mode for the number of degrees of freedom $\nu$ under the uniform prior, for different sample sizes and based on a Gibbs sampler of length $M=1000$ after a burn-in period of $M_{0}$ draws. Boxplots are based on $R=50$ datasets. 

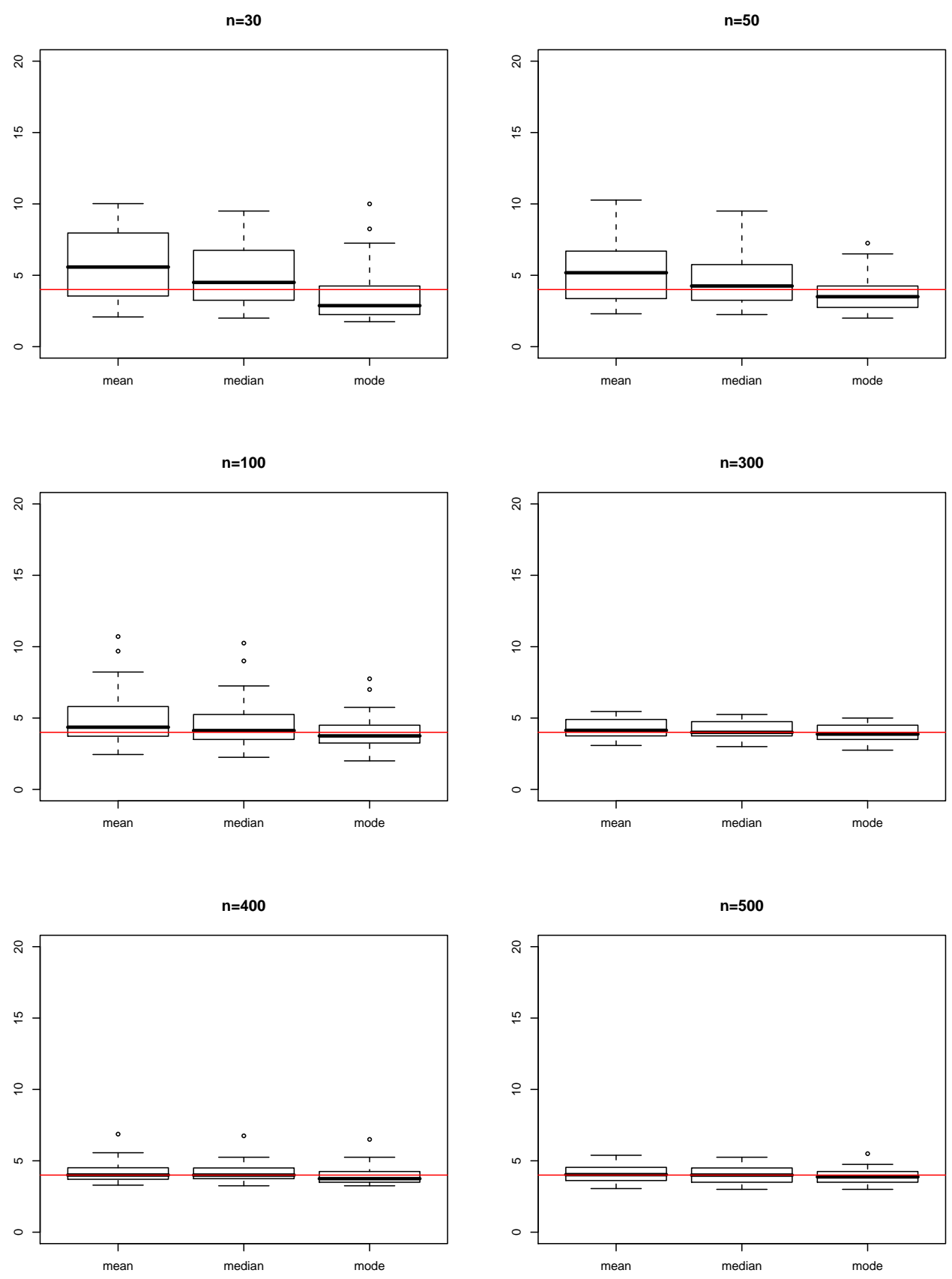

Figure 6: i.i.d. model. Posterior mean, median and mode for the number of degrees of freedom $\nu$ under the Jeffreys prior, for different sample sizes and based on a Gibbs sampler of length $M=1000$ after a burn-in period of $M_{0}$ draws.Boxplots are based on $R=50$ datasets. 

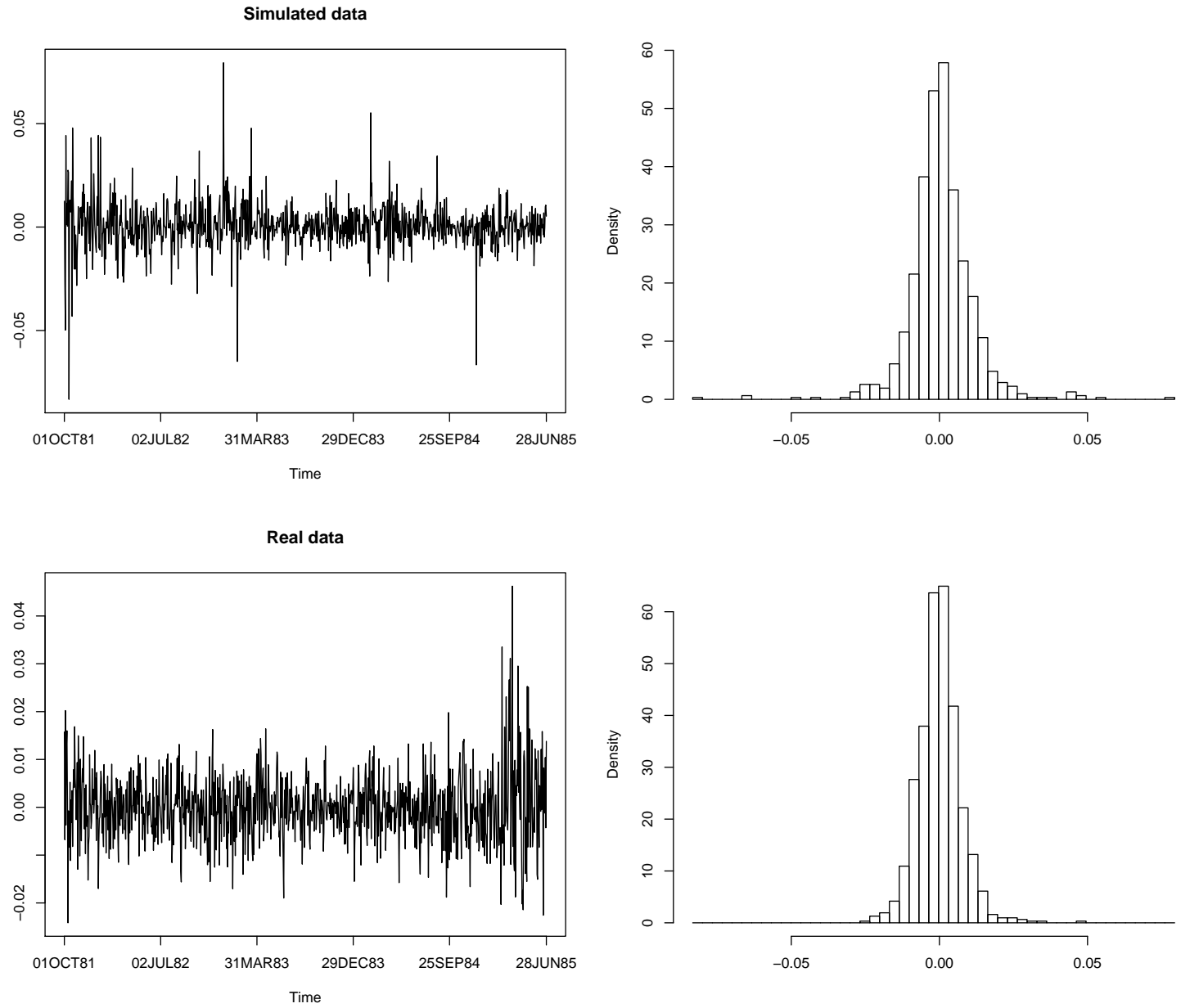

Figure 7: $S V$ - $t$ model. The top row corresponds to simulated data $(T=937)$ from the SV- $t_{\nu}$ model with parameters $\nu=4, \alpha=-0.202, \beta=0.980, \tau^{2}=0.018$ and $x_{0}=$ -8.053. The bottom row corresponds to JPR's (1994) British Pound vs US Dollar exchange $(T=937)$ daily rates from go from October 1st,1981 to June 28th,1985. 


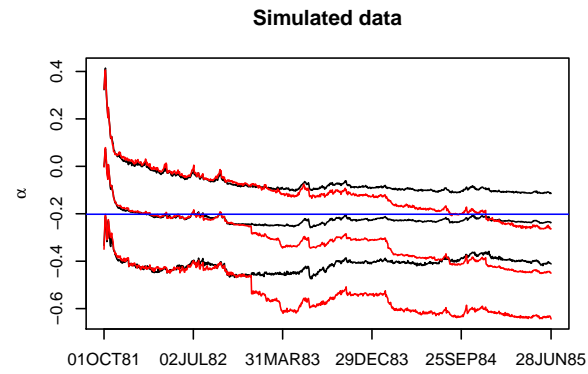

Time



Time

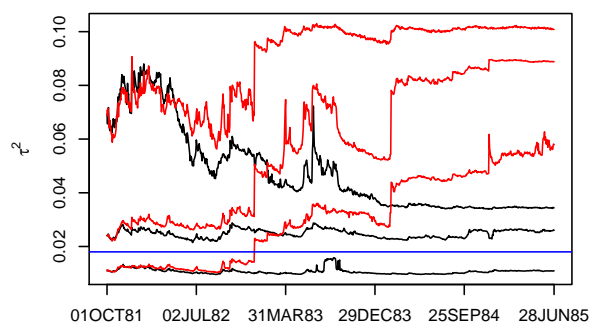

Time

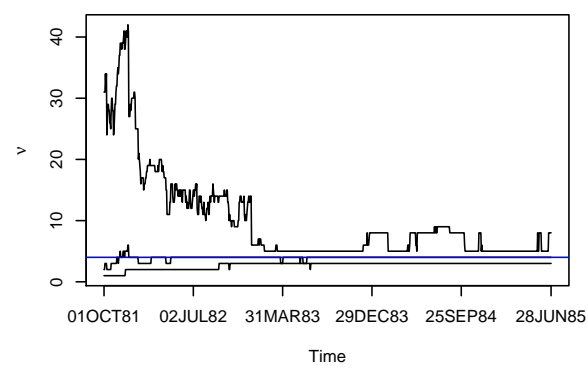

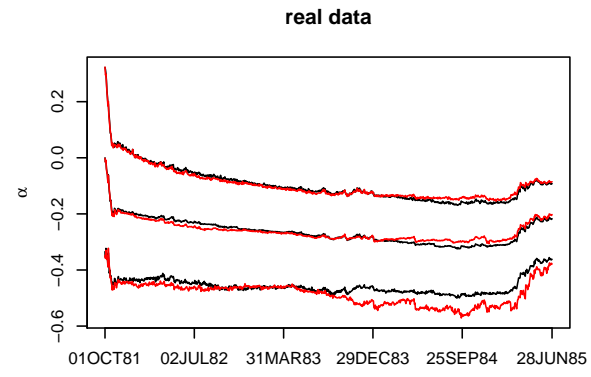

Time

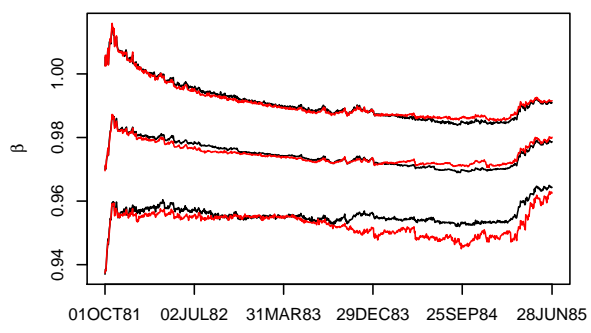

Time

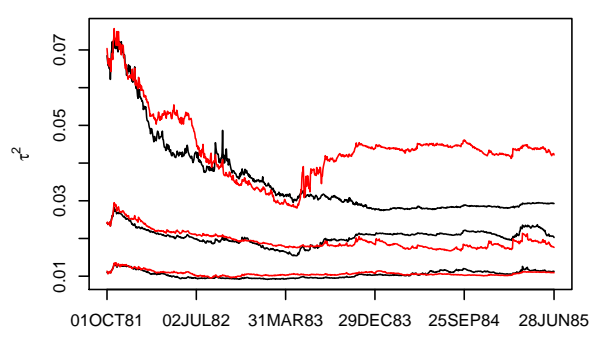

Time

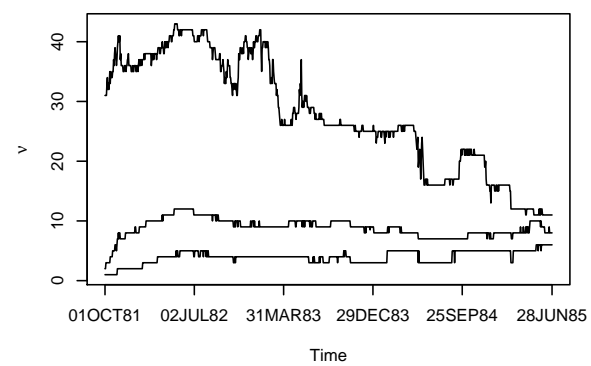

Figure 8: SV-t model. $(2.5,50,97.5)$-th percentiles of the sequential marginal posterior distributions of $\alpha, \beta, \tau^{2}$ and $\nu$ for the normal (red lines) and Student's $t$ (black lines) models. 

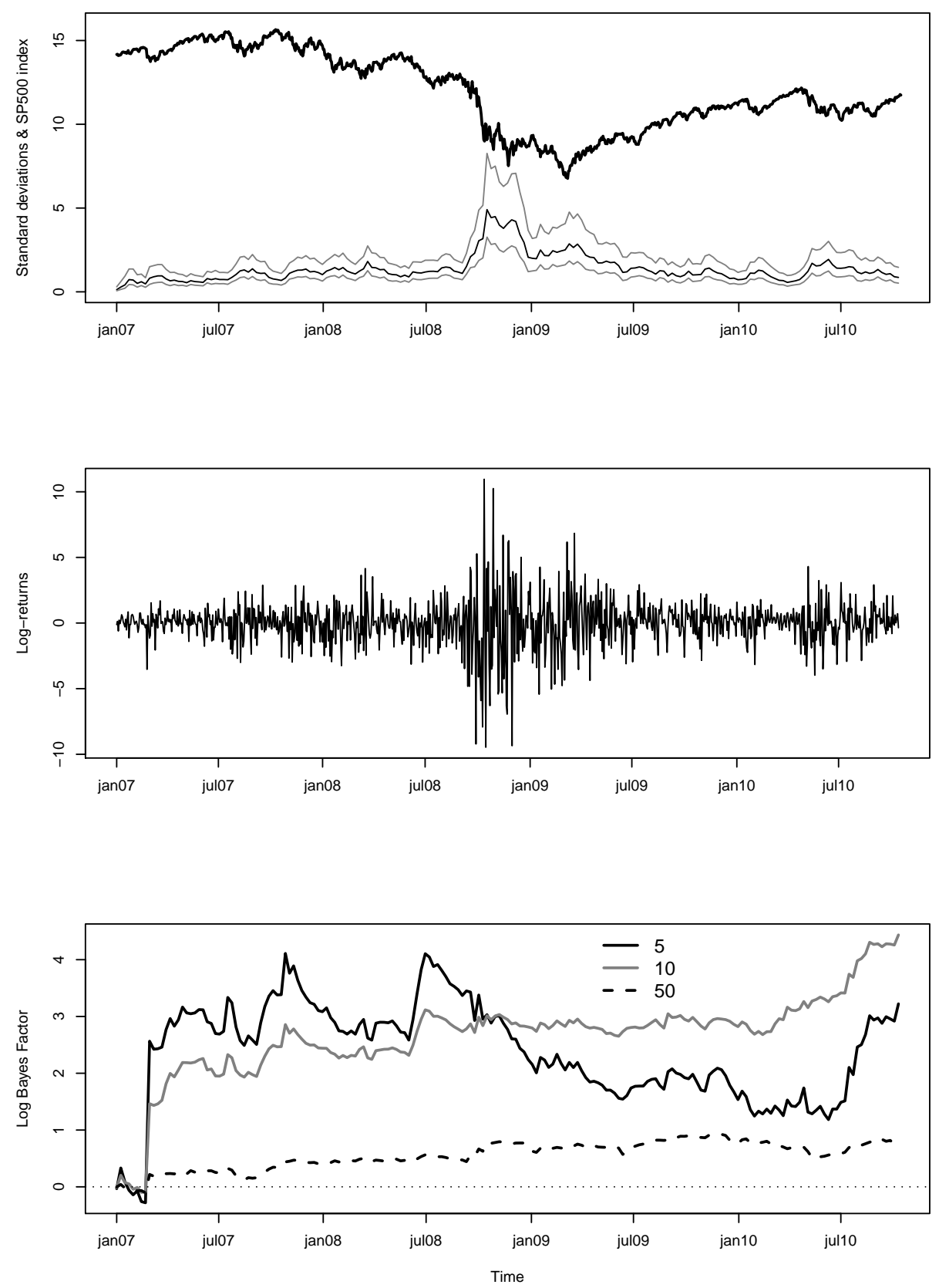

Figure 9: SV-t model for S\&P500 returns. Top frame: S\&P500 daily closing price (divided by 100: solid thick line) along with PL approximations to the $(2.5,50,97.5)$ th percentiles of the posterior distributions of the time-varying standard deviations $p\left(\exp \left\{x_{t} / 2\right\} \mid y^{t}\right)$, for $t=1, \ldots, T$, under the SV- $t_{10}$ model. Middle frame: Log returns. Bottom frame: Logarithm of the Bayes factors of $t_{\nu}$ against normality for $\nu \in\{5,10,50\}$. 